Bilateral dentigerous cysts related to third molar teeth are very rare

The treatment of choice is usually surgical enucleation

However, in this case due to loss of follow up, spontaneous regression of both cysts occurred

and this is the first reported case.

\title{
Spontaneous regression of bilateral dentigerous cysts associated with impacted mandibular third molars
}

\author{
N. Shah, ${ }^{1}$ H. Thuau ${ }^{2}$ and T. Beale ${ }^{3}$
}

A case is described where a routine panoramic radiograph revealed radiolucencies, compatible with dentigerous cysts, associated with the crowns of bilateral impacted lower third molars. Both cysts had regressed in a follow up radiograph three years later with no intervention. This is the first reported case of spontaneous regression of bilateral dentigerous cysts.

The dentigerous cyst is the second most common odontogenic cyst and originates from reduced enamel epithelium after the crown of the tooth has begun to form. It is most prevalent in the third molar region and is therefore a common cause of a radiolucency associated with the crown of an impacted third molar. ${ }^{1}$

Dentigerous cysts are usually solitary. Bilateral or multiple dentigerous cysts are rare, mainly found in association with syndromes. There have been 12 reported cases of bilateral dentigerous cysts and only six affecting the third molars. ${ }^{2}$ The treatment of choice is usually enucleation of the cyst with removal of the tooth if necessary. Sometimes, marsupialisation can be carried out if the cyst is too large. There have been a few reports of spontaneous regression of a dentigerous cyst. ${ }^{3-5}$

We present the seventh case of bilateral dentigerous cysts affecting the third molars but the first report of spontaneous regression of bilateral dentigerous cysts.

${ }^{1 *}$ Staff Grade, Department of Oral and Maxillofacial Surgery, Central Middlesex Hospital; ${ }^{2}$ Consultant, Department of Oral and Maxillofacial Surgery, Central Middlesex Hospital; ${ }^{3}$ Consultant, Department of Radiology, Royal National ENT Hospital

"Correspondence to: Dr Nihir Shah, Department of Oral and Maxillofacial Surgery and Department of Radiology, Central Middlesex Hospital, Acton Lane, London, NW10 7NS

\section{Refereed Paper}

Received 19.06.00; Accepted 12.09.00

๑ British Dental Journal 2002; 192: 75-76

\section{CASE REPORT}

A 39 year old Asian patient was referred by his general dental practitioner in May 1996 complaining of pain and clicking from his temporomandibular joints with severe temporal headaches. There was no history of trauma and he had no symptoms from the third molars. His medical history was unremarkable.

Clinical examination revealed a click from both temporomandibular joints on opening but with no limitation or deviation of the mandible. Intraorally, the lower third molars were unerupted. A routine panoramic radiograph (Fig 1) revealed two horizontally impacted lower third molars. Both teeth were associated with a well defined unilocular radiolucency, approximately $1.5 \mathrm{cms}$ in diameter, encompassing the crowns and attached to the amelocemental junction. On both sides the radiolucencies extended to the mesial aspect of the lower second molars, inferiorly to within $2 \mathrm{~mm}$ of the lower border of the mandible and superiorly to just below the alveolar crest. There was no displacement of the teeth and there was no apparent resorption of the roots of the lower second molars.

As there were no symptoms arising from these third molars, the patient was treated for his temporomandibular disorder with a view to reassessing the third molars at a later date. Unfortunately, he was lost to follow up but was re-referred in
February 1999 with a recurrence of his temporomandibular symptoms. Again, there was no history of symptoms from the third molars.

Clinical examination revealed no limitation or deviation of the mandible on opening. There was tenderness from the right temporomandibular joint on palpation. Intraorally, there were wear facets evident on all the teeth and the lower third molars were still unerupted. A follow up panoramic radiograph (Fig 2) showed apparent bony infill of the previous radiolucency associated with both lower third molars. There had been no eruption of the third molars but a slight mesial movement was noted.

The patient's temporomandibular symptoms were managed conservatively and he is currently on regular review.

\section{DISCUSSION}

The dentigerous cyst is usually associated with third molars and envelops all or part of the crown from its attachment at the amelocemental junction. Radiographically they are classically seen as radiolucent shadows associated with unerupted teeth and seemingly attached to the amelocemental junction. ${ }^{1}$ The final diagnosis can only be confirmed histologically after surgical removal of the cyst.

The case reported above fits well with the classic radiographic description of a 
dentigerous cyst. However, in the absence of surgical and histological confirmation, the differential diagnoses would also include odontogenic keratocysts or ameloblastoma.

Spontaneous resolution does occur ${ }^{3-5}$ but the above case is interesting due to regression of both dentigerous cysts. It is difficult to explain how this may have occurred as there was no history of pericoronitis and the patient did not have any dental treatment in the intervening period. Presumably, there was a communication to the oral cavity somehow leading to disruption of the cyst wall and marsupialisation.

1. JV Soames, J C Southam. Oral Pathology, 2nd ed. pp74-76. Oxford: Oxford University Press, 1993.

2. KS Ko, D G Dover, R C K Jordan. Bilateral Dentigerous Cysts- Report of an unusual case and review of the literature. J Can Dent Assoc 1999; 65: 49-51.

3. A M Adams, A G Walton. Case Report. Spontaneous regression of a radiolucency associated with an ectopic third molar. Dentomaxillofac Radiol 1996; 25: 162-164.

4. SPIrving, M D Winston-Salem. Spontaneous regression of a dentigerous cyst in a middle-aged adult Oral Surg 1984: 57:604-605.

5. G L Freedman. A Disappearing Dentigerous Cyst: Report of a Case. J Oral Maxillofac Surg 1988; 46 885-886.

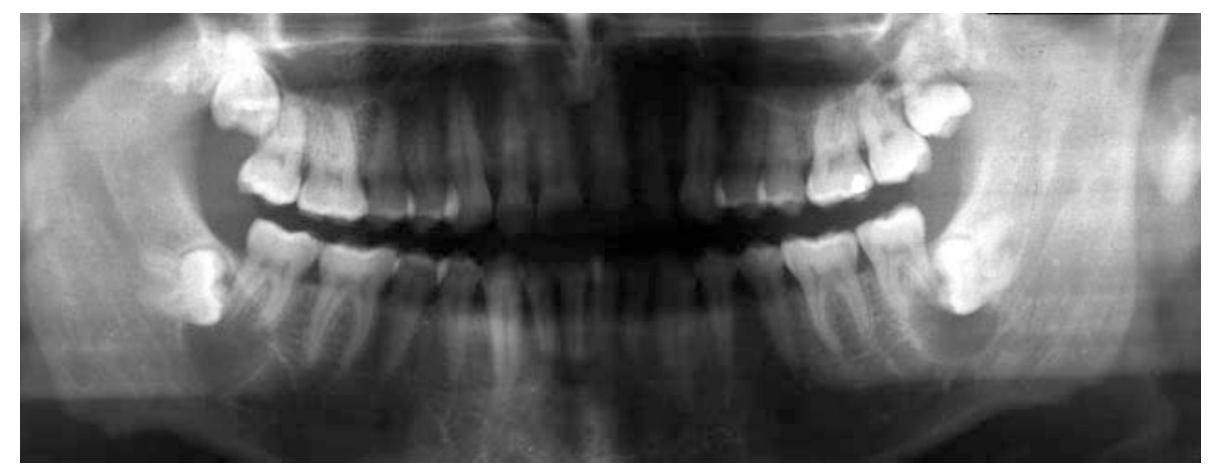

Figure 1. Panoramic radiograph showing radiolucencies, compatible with dentigerous cysts, associated with both horizontally impacted mandibular third molars.

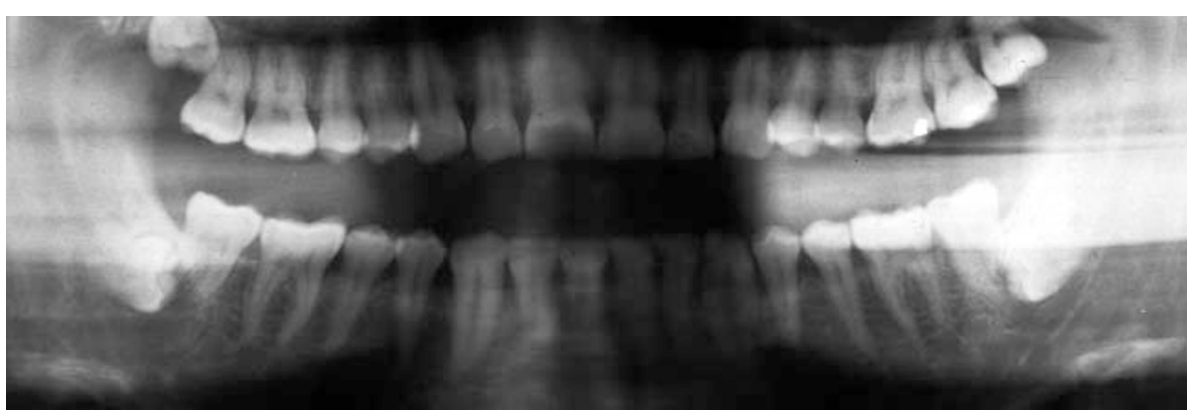

Figure 2. Follow up radiograph three years later showing spontaneous regression of the cysts associated with both mandibular third molars. 